\title{
Search for Heavy Stable Charged Particles in the CMS Experiment using the RPC Phase II upgraded detectors
}

To cite this article: G. Ramirez-Sanchez et al 2019 JINST 14 C11011

View the article online for updates and enhancements.

\section{IOP ebooks}

Bringing together innovative digital publishing with leading authors from the global scientific community. Start exploring the collection-download the first chapter of every title for free. 


\section{Search for Heavy Stable Charged Particles in the CMS Experiment using the RPC Phase II upgraded detectors}
G. Ramirez-Sanchez, ${ }^{w, 1}$ A. Fagot, ${ }^{a}$ M. Gul, ${ }^{a}$ C. Roskas,,${ }^{a}$ M. Tytgat, ${ }^{a}$ N. Zaganidis, ${ }^{a}$
S. Fonseca De Souza, ${ }^{b}$ A. Santoro, ${ }^{b}$ F. Torres Da Silva De Araujo, ${ }^{b}$ A. Aleksandrov, ${ }^{c}$
R. Hadjiiska, ${ }^{c}$ P. laydjiev, ${ }^{c}$ M. Rodozov, ${ }^{c}$ M. Shopova, ${ }^{c}$ G. Sultanov, ${ }^{c}$ A. Dimitrov, ${ }^{d}$ L. Litov, ${ }^{d}$
B. Pavlov, ${ }^{d}$ P. Petkov ${ }^{d}$ A. Petrov,${ }^{d}$ S.J. Qian, ${ }^{e}$ D. Han, ${ }^{f}$ W. Yi, ${ }^{f}$ C. Avila, ${ }^{g}$ A. Cabrera,${ }^{g}$
C. Carrillo, ${ }^{g}$ M. Segura, ${ }^{g}$ S. Aly, ${ }^{h}$ Y. Assran, ${ }^{h}$ A. Mahrous, ${ }^{h}$ A. Mohamed, ${ }^{h}$ C. Combaret,${ }^{i}$
M. Gouzevitch, ${ }^{i}$ G. Grenier, ${ }^{i}$ F. Lagarde,${ }^{i}$ I.B. Laktineh, ${ }^{i}$ H. Mathez,${ }^{i}$ L. Mirabito, ${ }^{i}$
K. Shchablo, ${ }^{i}$ I. Bagaturia, ${ }^{j}$ D. Lomidze,${ }^{j}$ I. Lomidze,${ }^{j}$ L.M. Pant, ${ }^{k}$ V. Bhatnagar, ${ }^{l}$ R. Gupta, ${ }^{l}$
R. Kumari, ${ }^{l}$ M. Lohan, ${ }^{l}$ J.B.Singh, ${ }^{l}$ V. Amoozegar, ${ }^{m}$ B. Boghrati, ${ }^{m, n}$ H. Ghasemy, ${ }^{m}$
S. Malmir ${ }^{m}$ M. Mohammadi Najafabadi, ${ }^{m}$ M. Abbrescia, ${ }^{o}$ A. Gelmi, ${ }^{o}$ G. laselli, ${ }^{o}$ S. Lezki, ${ }^{o}$
G. Pugliese,${ }^{o}$ L. Benussi, ${ }^{p}$ S. Bianco, ${ }^{p}$ D. Piccolo, ${ }^{p}$ F. Primavera, ${ }^{p}$ S. Buontempo, ${ }^{q}$
A. Crescenzo, ${ }^{q}$ G. Galati, ${ }^{q}$ F. Fienga, ${ }^{q}{ }^{\text {I. Orso }},{ }^{q}$ L. Lista, ${ }^{q}$ S. Meola, ${ }^{q}$ P. Paolucci, ${ }^{q}$
E. Voevodina, ${ }^{q}$ A. Braghieri, ${ }^{r}$ P. Montagna,${ }^{r}$ M. Ressegotti, ${ }^{r}$ C. Riccardi, ${ }^{r}$ P. Salvini, ${ }^{r}$
P. Vitulo, ${ }^{r}$ S. W. Cho, ${ }^{s}$ S. Y. Choi, ${ }^{s}$ B. Hong, ${ }^{s}$ K. S. Lee, ${ }^{s}$ J. H. Lim,${ }^{s}$ S. K. Park, ${ }^{s}$ J. Goh, ${ }^{t, a} a$
T.J. Kim, ${ }^{t}$ S. Carrillo Moreno, ${ }^{u}$ O. Miguel Colin, ${ }^{u}$ F. Vazquez Valencia, ${ }^{u}$
S. Carpinteyro Bernardino, ${ }^{v}$ J. Eysermans, ${ }^{v}$ I. Pedraza, ${ }^{v}$ C. Uribe Estrada, ${ }^{\nu}$
R. Reyes-Almanza, ${ }^{w}$ M.C. Duran-Osuna, ${ }^{w}$ M. Ramirez-Garcia, ${ }^{w}$ A. Sanchez-Hernandez, ${ }^{w}$ R.I. Rabadan-Trejo, ${ }^{w}$ H. Castilla-Valdez, ${ }^{w}$ A. Radi, ${ }^{x}$ H. Hoorani, ${ }^{y}$ S. Muhammad, ${ }^{y}$ M.A. Shah ${ }^{y}$ and I. Crotty ${ }^{z}$ on behalf of the CMS collaboration

\footnotetext{
${ }^{a}$ Ghent University, Dept. of Physics and Astronomy, Proeftuinstraat 86, B-9000 Ghent, Belgium

${ }^{b}$ Dep. de Fisica Nuclear e Altas Energias, Instituto de Fisica, Universidade do Estado do Rio de Janeiro, Rua Sao Francisco Xavier, 524, BR - Rio de Janeiro 20559-900, RJ, Brazil

${ }^{c}$ Bulgarian Academy of Sciences, Inst. for Nucl. Res. and Nucl. Energy,

Tzarigradsko shaussee Boulevard 72, BG-1784 Sofia, Bulgaria

${ }^{d}$ Faculty of Physics, University of Sofia, 5 James Bourchier Boulevard, BG-1164 Sofia, Bulgaria

${ }^{e}$ School of Physics, Peking University, Beijing 100871, China

${ }^{f}$ Tsinghua University, Shuangqing Rd, Haidian Qu, Beijing, China

${ }^{g}$ Universidad de Los Andes, Apartado Aereo 4976, Carrera 1E, no. 18A 10, CO-Bogota, Colombia
}

${ }^{1}$ Corresponding author. 
${ }^{h}$ Egyptian Network for High Energy Physics, Academy of Scientific Research and Technology,

101 Kasr El-Einy St. Cairo Egypt

${ }^{i}$ Universite de Lyon, Universite Claude Bernard Lyon 1, CNRS-IN2P3,

Institut de Physique Nucleaire de Lyon,

Villeurbanne, France

${ }^{j}$ Georgian Technical University, 77 Kostava Str., Tbilisi 0175, Georgia

${ }^{k}$ Nuclear Physics Division Bhabha Atomic Research Centre Mumbai 400 085, India

${ }^{l}$ Department of Physics, Panjab University, Chandigarh Mandir 160 014, India

${ }^{m}$ School of Particles and Accelerators, Institute for Research in Fundamental Sciences (IPM), Tehran, Iran

${ }^{n}$ School of Engineering, Damghan University, Damghan, Iran

${ }^{o}$ INFN, Sezione di Bari, Via Orabona 4, IT-70126 Bari, Italy

${ }^{p}$ INFN, Laboratori Nazionali di Frascati (LNF), Via Enrico Fermi 40, IT-00044 Frascati, Italy

q INFN, Sezione di Napoli, Complesso Univ. Monte S. Angelo, Via Cintia, IT-80126 Napoli, Italy

${ }^{r}$ INFN, Sezione di Pavia, Via Bassi 6, IT-Pavia, Italy

${ }^{s}$ Korea University, Department of Physics, 145 Anam-ro, Seongbuk-gu, Seoul 02841, Republic of Korea

${ }^{t}$ Hanyang University, 222 Wangsimni-ro, Sageun-dong, Seongdong-gu, Seoul, Republic of Korea

${ }^{u}$ Universidad Iberoamericana, Mexico City, Mexico

${ }^{v}$ Benemerita Universidad Autonoma de Puebla, Puebla, Mexico

${ }^{w}$ Cinvestav,

Av. Instituto Politécnico Nacional No. 2508, Colonia San Pedro Zacatenco, CP 07360,

Ciudad de Mexico D.F., Mexico

${ }^{x}$ Sultan Qaboos University, Al Khoudh,Muscat 123, Oman

${ }^{y}$ National Centre for Physics, Quaid-i-Azam University, Islamabad, Pakistan

${ }^{z}$ Dept. of Physics, Wisconsin University, Madison, WI 53706, United States

${ }^{a}$ Kyunghee University, 26 Kyungheedae-ro, Hoegi-dong, Dongdaemun-gu, Seoul, Republic of Korea

E-mail: gramirez@fis.cinvestav.mx

AвSTRACT: Several theoretical models inspired by the idea of supersymmetry (SUSY) accommodate the possibility of Heavy Stable Charged Particles (HSCPs). The Phase II upgrade of the CMS-RPC system will allow the trigger and identification of this kind of particles exploiting the Time-of-Flight Technique with the improved time resolution that a new Data Acquisition System (DAQ) system will provide ( $\sim 2 \mathrm{~ns})$. Moreover, new Resistive Plate Chambers (RPC) detector chambers will be installed to extend the acceptance coverage up to $|\eta|<2.4$ with similar time resolution and better spatial resolution.

We present a trigger strategy to detect HSCPs with the RPC detectors. Its performance is studied with Monte Carlo simulations and the expected results with the High Luminosity Large Hadron Collider (HL-LHC) data are shown.

KEYwORDS: Gaseous detectors; Resistive-plate chambers 


\section{Contents}

1 Introduction 1

2 RPC detector Phase II upgrade 2

3 Time-of-flight technique 3

4 Efficiency 4

5 Conclusion 5

\section{Introduction}

In 2026 the current Large Hadron Collider (LHC) will turn into the High Luminosity LHC (HLLHC) due to the increase of about ten times the luminosity and energy at the center of mass for $p-p$ collisions will be increased to $14 \mathrm{TeV}$. This is with the purpose of continued studying and testing of the Standard Model (SM) but especially to explore different scenarios of physics Beyond the Standard Model (BSM), including supersymmetry (SUSY) and heavy gauge bosons.

To fully exploit what the new capabilities HL-LHC will provide, an upgrade will take place in 2023, the Link System of the complete Resistive Plate Chambers (RPC) detector will be improved with the addition of more robust and up-to-date components. This improvement in the electronics will enable the Link System to attain the time intrinsic resolution of the RPC detector of the order of $1 \mathrm{~ns}$. In addition, new detectors will be installed, the so-called improved RPC (iRPC) that will extend the reach of the RPC detector and will have even better intrinsic resolution than the present system.

The improvement in the time resolution will be beneficial to achieve the requirements on trigger imposed by the increase in the luminosity. It will also allow the creation of a new trigger for the identification of slowly moving particles by measuring their Time-of-Flight on all the RPC stations with good precision. The speed of muon-like particles and the time (bunch crossing) of their origin will be computed with a fast algorithm to be implemented in the Phase II Level 1 trigger.

There are several theoretical models, which predict new massive particles stable enough to pass through the whole detector in the collider experiments. Supersymmetry is one of the theoretical models which can explain the existence of the dark matter and problems in the Standard Model (SM) by introducing a new symmetry called R-parity. Partners of the existing particles in the SM are expected from the model including the Lightest Supersymmetric Particle (LSP), which must be stable. The Universal Extra Dimension (UED) model also predicts similar type of particles. There are other theoretical scenarios with parity-like symmetries, magnetic monopoles [2]. Some of them are expected to be non-neutral such that they have electromagnetic or strong interactions with the detectors along their trajectory with small velocity. One of the simplest scenarios is pairproduced supersymmetric $\tau$ leptons $(\tilde{\tau})$, which only interacts via the electromagnetic interaction. 
A slowly-moving muon-like track can be reconstructed as a result of propagation of this type of HSCP. For particles with $\beta=v / c<0.6$, the Time-of-Flight at the muon detectors would be more than $25 \mathrm{~ns}$ and the information can be ignored in the standard trigger algorithms by assigning them to a different bunch crossing. In this study, we consider the $\tilde{\tau}$ pair from the proton-proton collisions as a benchmark point.

Standard muon trigger algorithms may not be optimal for the HSCP searches in this perspective, therefore a new trigger algorithm has to be developed.

\section{RPC detector Phase II upgrade}

The CMS detector is formed of different subdetectors and a superconductor solenoid of 3.8 T. A schematic visualization of its quadrants is presented in figure 1 . The pixel detector is found in the innermost part next to the beam pipe, followed by the Silicon Tracking detector, the electromagnetic and hadronic calorimeters and surrounded by the solenoid. The muon system is situated in the outermost part the detector and is formed by three sub-detectors that work based on different technologies. These detectors are the Cathode Strip Chambers (CSC), the Drift Tubes (DT) and RPC detectors. A detailed description of the detector can be found in [1].

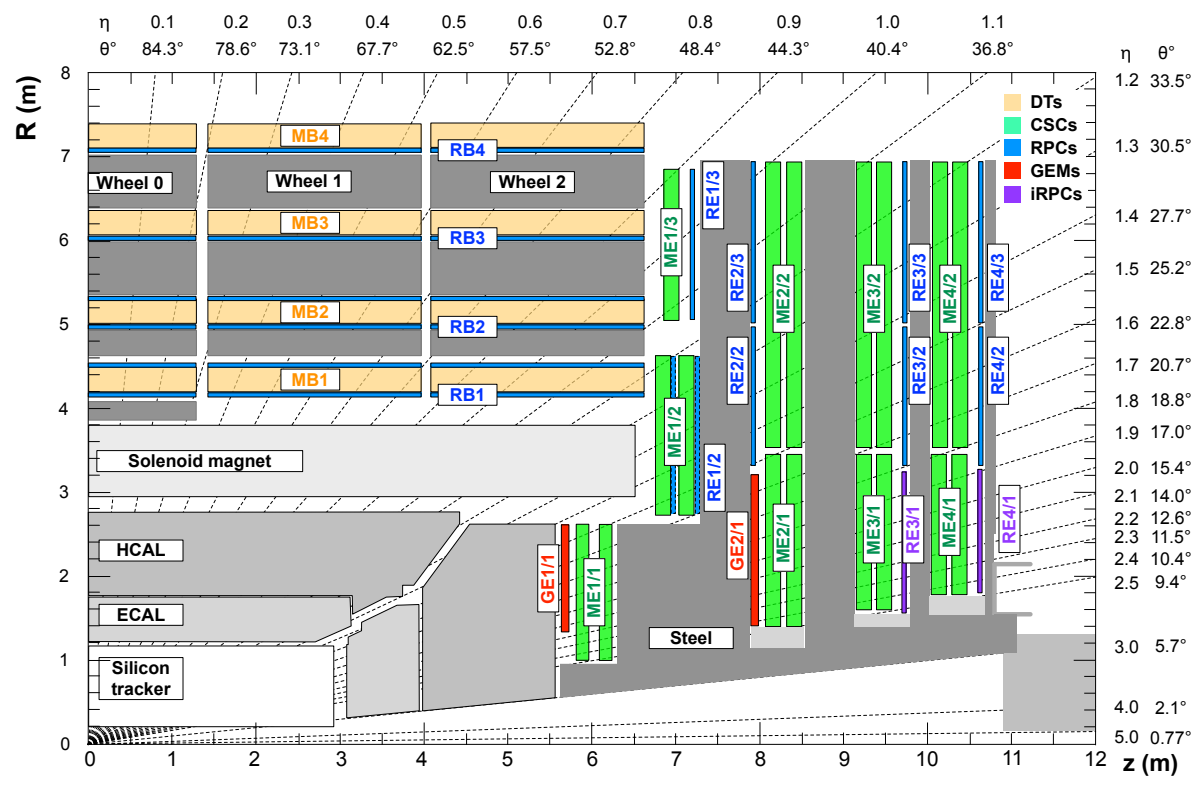

Figure 1. The RPC detector is represented together with the other sub-detector of CMS. The present RPC system is represented in blue, whereas the iRPC is represented in purple. RB and RE denote RPC Barrel and RPC Endcap respectively.

In the present work we are only concerned about the RPC detector. Currently it covers a pseudorapidity $(\eta)$ up to 1.9 and possesses an intrinsic time resolution of the order of $1.5 \mathrm{~ns}$, as shown in figure 2, but the Link System records the RPC hits information in steps of one Bunch Crossing BX $(25 \mathrm{~ns})$, thus losing the full timing resolution of the detector apart from the mere bunch-crossing identification. 


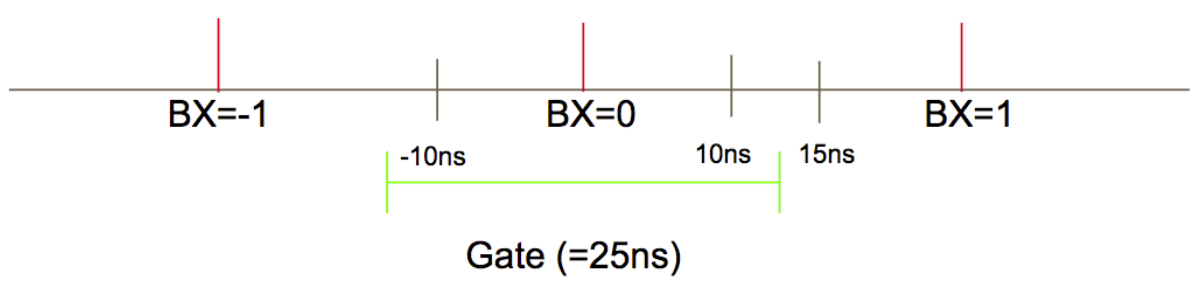

Figure 2. Diagram of bunch crossing assignment performed by the RPC detector, with the current conditions.

The Link System of the RPC connects the detector and the trigger processors. It records bunch crossing (BX) with very high precision, with help of the good intrinsic time resolution of the RPC detectors as in the figure 2. Full timing resolution can be recovered with the new Link Boards which will be based on a faster FPGAs.

The RE3/1 and RE4/1 are detectors that were included in the original design, but not installed due to financial reasons. They will be added as part of the upgrade. Their implementation will improve stand-alone (using only the RPC detector) measurements and help to decrease the background rates. These new detectors will make two measurements (each at every end of the strips) thus improving the spatial and time resolutions. In particular, they will improve the time resolution precision by more than two times with respect to using CSC alone.

\section{Time-of-flight technique}

We developed a new trigger algorithm to detect HSCP using the Time-of-Flight (ToF) of the HSCP at the RPC detectors. In the algorithm we propose, we measure the speed of the particle by using the timing and position information of the RPC hits within a cone $\Delta R=\sqrt{\Delta \eta^{2}+\Delta \phi^{2}}<0.3$ around the generated particle as shown in the figure 4 . We perform a linear fit on the ToF and the distance from the origin, $r$. Let us note first that the detector does not register the speed of a particle, it measures instead its relative speed with respect to a particle moving at light speed. For this reason, muons are expected to be measured at around zero as it can be seen in figure 3 , and because of experimental error they could also have negative speed. The distribution of HSCPs is expected to be displaced, with respect to distribution for a muon, slightly to the right due to difference of speed. This property is exploited with the new algorithm to distinguish the HSCPs from the muons.

In order to perform measurement of $\beta$ and $t_{0}$ we first have to do the matching of the RPC hits with the generated particles.

A schematic view of how the muons and HSCP would be seen by the detector can be found in figure 5. The vertical axis represents the time measured by the RPC detector in each of the chambers the particle travels through. On the other hand, the horizontal axis is the distance from the collision point to the position where the particle is detected measured by RPC detector. The diagram shows three successive bunch crossings, two of which contain muons represented as horizontal lines. The diagram also shows the RPC time measurements from two HSCPs having slopes different from zero due to their speed significantly slower than light. 
HSCPs can have hits spanning two or more BXs. The time delay $\Delta t$ is related to the speed $v$ of a particle, starting from the simple equation:

$$
v_{\text {layer }}=\frac{d}{T o F t}
$$

where $v_{\text {layer }}, d$ and ToF are the mean speed, the distance and the Time-of-Flight from the interaction point to the detection point, respectively. After some algebra we have found that $\beta$ and $t_{0}$ are related by the following expression containing the speed of the particle:

$$
t_{\text {delay }}=t_{0}+\frac{d}{c}\left(\beta^{-1}-1\right),
$$

where $t_{\text {delay }}$ is the time detected, $t_{0}$ is the time expected for a particle at the Bunch Crossing $0, c$ is the speed of light and $\beta$ is the speed of the particle. A penetrating charged particle leaves a trail of hits in RPC chambers along its trajectory. The Time-of-Flight can be computed in each RPC station with respect to a number of BX hypotheses. From this we can distinguish 4 specific scenarios:

- Muon on time. It will be seen as a horizontal line with intersection on $B X=0$

- Muon out of time. It will be seen as a horizontal line with intersection on $B X \neq 0$

- HSCP on time. It will be seen as a diagonal line with intersection on $B X=0$

- HSCP out of time. It will be seen as a diagonal line with intersection on $B X \neq 0$

Should there be a common velocity solution, derived from eq. (3.2), with $\beta<0.6$, a trigger is formed.

Ordinary muon triggers are capable to handle particles at $\beta>0.6$ because delays are not too large. We require the new trigger to find a common velocity solution for $\beta<0.6$ to recover the trigger efficiency.

The performance of this algorithm has been studied in CMS full simulation. All the detector effects (electronics jitter, signal time propagation along strips) are taken into account. A particle speed measurement resolution is shown in figure 6 for the case of $25 \mathrm{~ns}$ signal sampling time (Phase I) and $1.56 \mathrm{~ns}$ sampling time provided with the upgraded RPC Link Board System.

The trigger algorithm is: at least 3 hits correlated in space, error in $\beta<30 \%$ (to assure good quality of the fit) and slope $>0$ (to exclude muons and identify slow moving particles)

\section{Efficiency}

The new trigger proposal will complement the present muon trigger whose efficiency sharply drops for particles with $\beta<0.6$. The efficiency of the RPC-HSCP algorithm as a function of $\beta$ is studied and compared with the standard L1 muon trigger. The results are shown in figure 7 The current CMS-HSCP Phase I trigger performs well down to $\beta \approx 0.75$.

The upgraded RPC Link Board System will allow us to trigger, at the correct BX, HSCPs with velocities as low as $\beta \sim 0.25$.

Possible improvements for this trigger proposal in the $\beta$ measurement could be achieved by matching the Tracker Track trigger to the HSCP muon trigger. The uncertainty coming from the 
propagation time along RPC strips can be reduced if the hit position is known along the local $y$ coordinate, or the global $\eta$. This correction is not needed for the iRPCs thanks to the two-end strip readout for these new detectors.

We concentrate on the performance in terms of specific HSCP parameters in a modelindependent way rather than providing an interpretation in a dedicated model. Given the wide range of new models, it is important to stay sensitive to a wide range of unusual signatures such as very slowly moving particles.

Figure 8 shows that a special HSCP-targeting L1 muon trigger, fully exploiting the improved timing of the upgraded RPC link system, can be efficient to HSCPs with velocities as low as $\beta \sim 0.2$ for the full $\eta$ region covered by the RPC detector, which would not be possible without the upgrade. Figure 9 shows the achievable mass resolution for a supersymmetric $\tilde{\tau}$ lepton of $1.6 \mathrm{TeV}$ mass.

\section{Conclusion}

The resolution for the HSCP mass obtained for Phase II at the trigger level is comparable to that realized in Run 2 studies based on offline time-of-flight information (using the DTs and CSCs). The information provided by the RPC trigger can be used as an independent crosscheck of the reconstructed mass.

The large gain in efficiency for very slowly moving particles in Phase II enabled by the upgrade of the RPC trigger can be exploited in a model independent HSCP search.

\section{Acknowledgments}

Special thanks go to Camilo Andres Carrillo Montoya, Junghwan John Goh and the group members of RPC HSCP for their valuable contributions to this work.

\section{References}

[1] M. Abbrescia et al., Local and global performance of double-gap resistive plate chambers operated in avalanche mode, Nucl. Instrum. Meth. A 434 (1999) 244.

[2] M. Fairbairn, A.C. Kraan, D.A. Milstead, T. Sjöstrand, P.Z. Skands and T. Sloan, Stable Massive Particles at Colliders, Phys. Rept. 438 (2007) 1 [hep-ph/0611040].

[3] G.F. Giudice and A. Romanino, Split supersymmetry, Nucl. Phys. B 699 (2004) 65 [Erratum ibid. B 706 (2005) 487-487] [hep-ph/0406088].

[4] N. Arkani-Hamed and S. Dimopoulos, Supersymmetric unification without low energy supersymmetry and signatures for fine-tuning at the LHC, JHEP 06 (2005) 073 [hep-th/0405159]. 


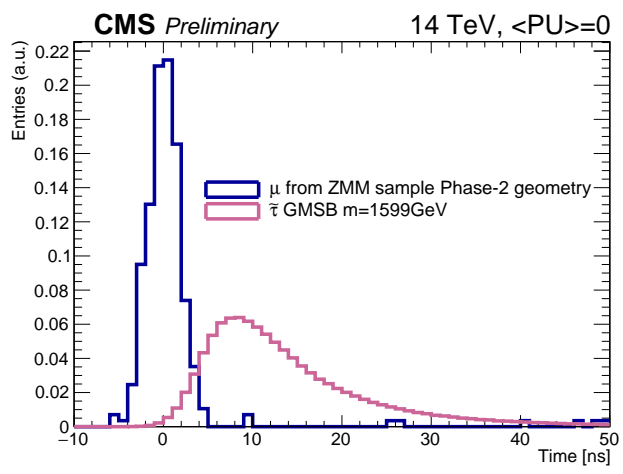

Figure 3. Simulated time distributions for muons and $\widetilde{\tau}$ s after the detector upgrade. Simulated RPC hit time distribution for muons from $Z \rightarrow \mu^{+} \mu^{-}$events and for semi-stable $\tilde{\tau}$ 's with $m \approx 1600 \mathrm{GeV}$, produced in $p p \rightarrow \overline{\tilde{\tau}}$ processes.

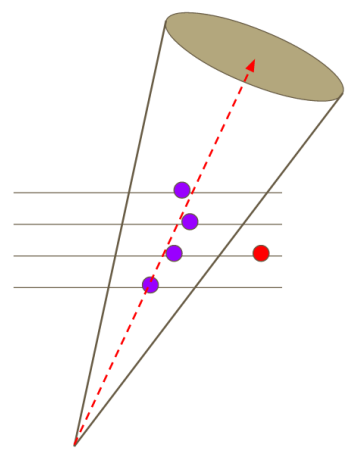

Figure 4. A cone (with $\Delta R<0.3$ ) is defined around the direction of the generated particle and only the RPC hits inside it are selected. 


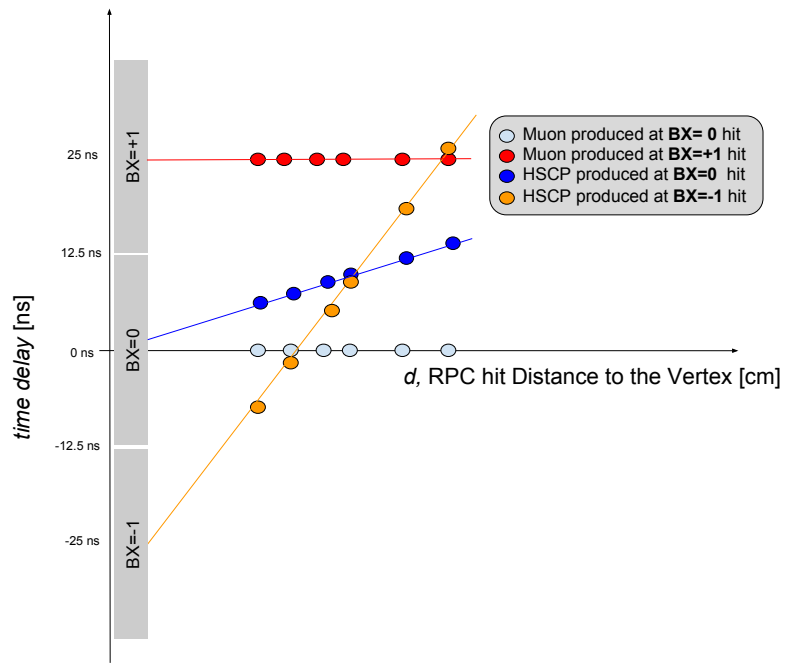

Figure 5. Diagram showing times measured at different RPC stations for particles originating at different BXs with different velocities. The $x$-axis represents the distances from IP to RPC detectors, while $y$-axis corresponds to time. Clock at all RPC stations is tuned so that particles moving with the speed of light are registered with the exact same "local" times. Hence, horizontal lines on this diagram represent relativistic particles.

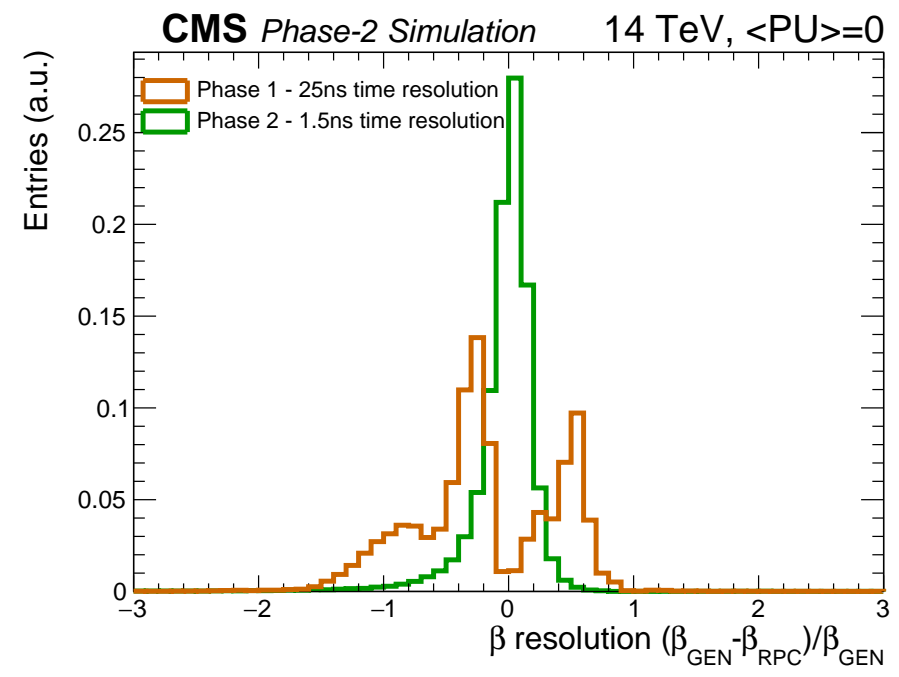

Figure 6. Speed resolution at L1 trigger level of a $\bar{\tau}$ particle (HSCP candidate) with $1.6 \mathrm{TeV}$ mass simulated with Pythia8 with Phase I and upgraded RPC Link Board System. 


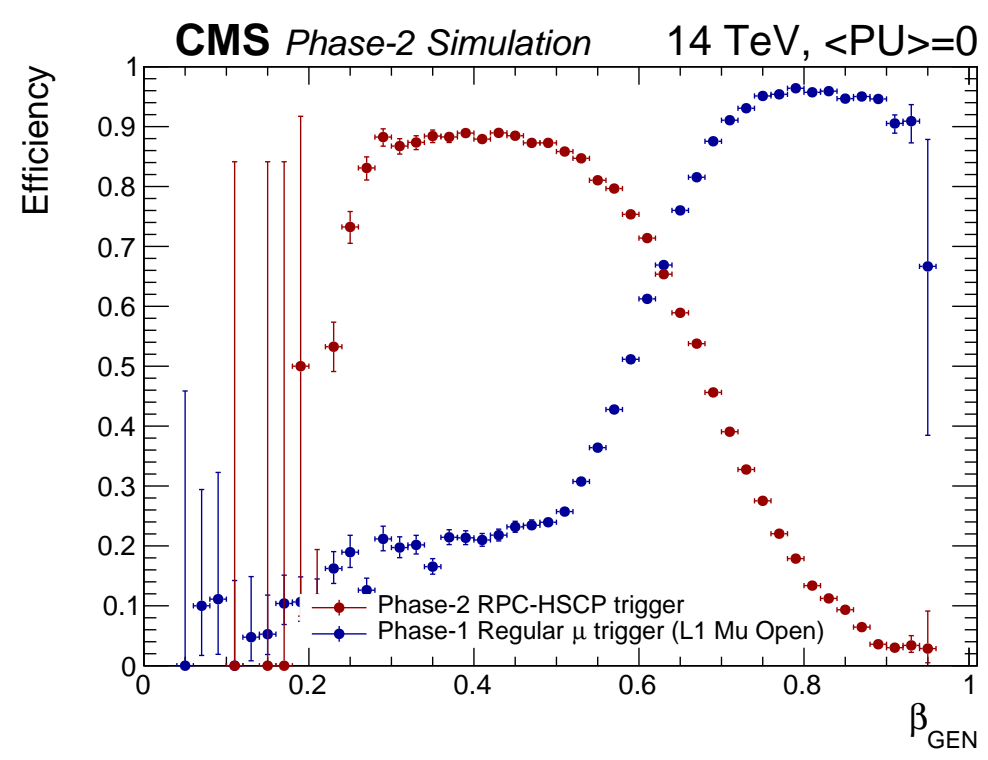

Figure 7. L1 Trigger efficiency as a function of an HSCP velocity $\beta$ for the 'regular' trigger (assuming the muon to come from the primary vertex, blue points) and a dedicated HSCP trigger, fully exploiting the improved timing of the upgraded RPC link system (red points).

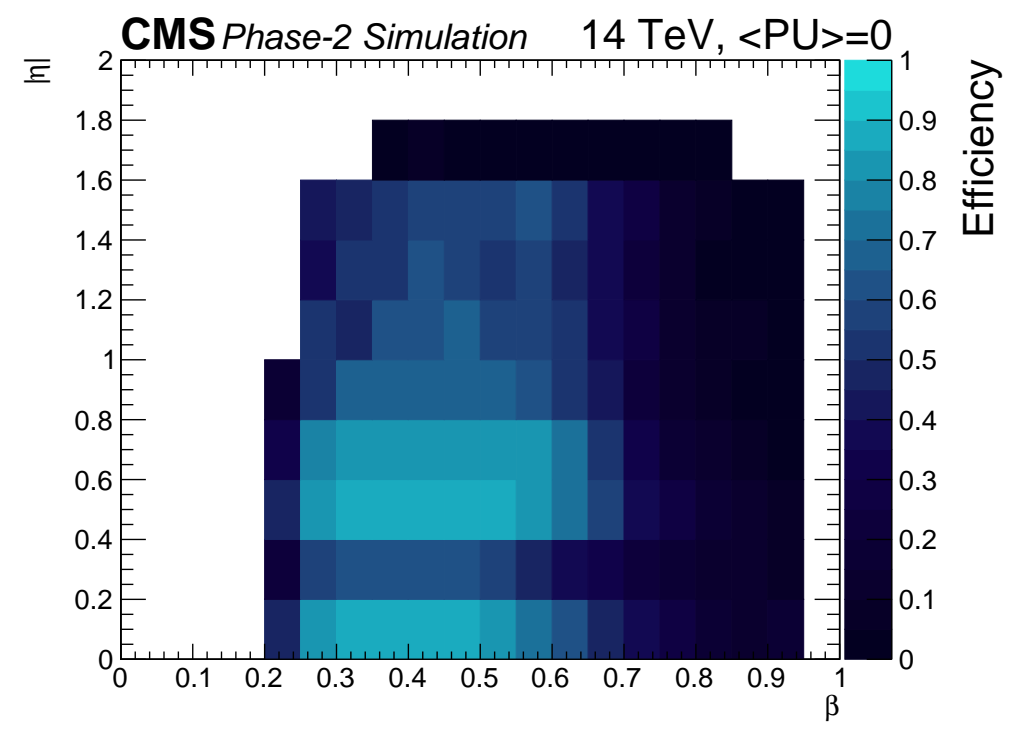

Figure 8. HSCP trigger efficiency as a function of HSCP velocity $\beta$ and pseudorapidity $\eta$. 


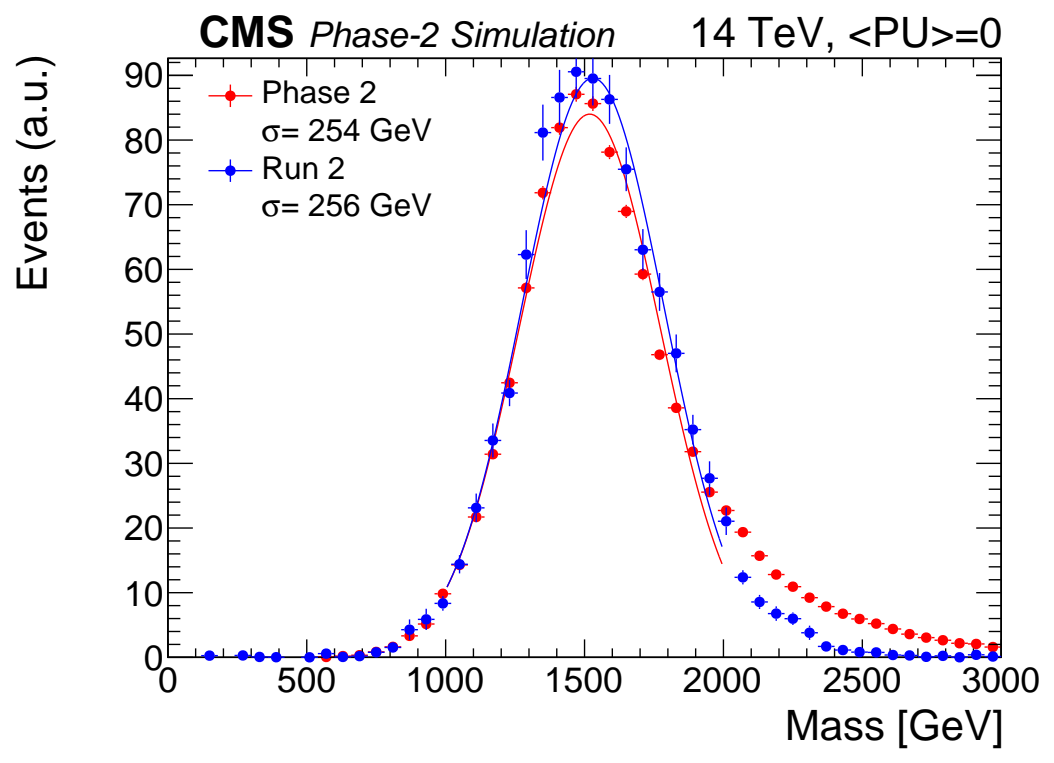

Figure 9. Comparison of mass resolution for a $1.6 \mathrm{TeV} \tilde{\tau}$. In Run 2 the shown resolution can only be achieved offline, while the upgraded RPC link-boards in Phase II provide a similar mass resolution already at Level 1 trigger level. 